\title{
El agua como tema transversal de actuación del derecho ambiental y sanitario en Brasil
}

\author{
The Water as a Transversal Theme of Action \\ of Environmental and Sanitary Law in Brazil
}

\section{Nicolau CARDoso NeTO*}

RESUMEN: La garantía de calidad de vida para la población brasileña involucra, sine qua non, acciones sobre la condición del medio ambiente. Las mismas pueden realizarse buscando la protección, prevención y mejoría de la condición ambiental - materias éstas del derecho ambiental- por medio de acciones que buscan identificar y detener los riesgos y divulgar las informaciones referentes a los factores ambientales que puedan causar enfermedades y otros agravios a la salud - acciones que competen al derecho sanitario-. Frente a tal constatación, el objeto de este estudio es analizar la problemática del agua como tema transversal de actuación en el derecho ambiental y sanitario. Este trabajo analizará el tema del agua por ser un tema común a dichos sistemas, con la intención de evaluar si las competencias y las estructuras del sistema ambiental y sanitario poseen composiciones diferentes actuando sobre el mismo asunto.

Palabras clave: derecho ambiental, derecho sanitario, agua.
ABSTRACT: The guarantee of quality of life for the Brazilian population involves, necessarily, actions on the condition of the environment. This performance can be achieved through actions which aim at protection, prevention and improvement of environmental condition - these competences belong to the branch of Environmental Law-, by means of actions that seek to identify and hold the risks and disseminate the information related to environmental factors that can cause diseases and other health problems - these competences belong to the branch of Health Law-. In view of this observation, is object of this study analyze water as crosscutting themes of action of Environmental and Health Law. To answer the problem, this paper will analyze a common theme between the two systems, the water, with the intention of evaluating if the competences and the structures of the environmental and sanitary system have different compositions acting on the same subject.

Keywords: Environmental Law, Health Law, Water.

* Doctor en derecho público por la UNISINOS, Brasil. Profesor de la Universidad FURB, Brasil. Integrante del Grupo de Estudios Derechos Fundamentales, Ciudadanía y Diferenciación. Correo: nicolau@scambiental.com.br; ORCID: 000-0002-9463-0673. 
SUMARIO: I. Introducción. II. Análisis de las ramas del derecho ambiental y sanitario. III. Conclusión. IV. Bibliografía.

\section{INTRODUCCIÓN}

El agua es, siempre fue y será un tema vigente para discutirse. La facilidad que la tecnología nos ofrece en la actualidad permite identificar nuevos factores a ser considerados para análisis; factores que pueden perjudicar los recursos hídricos como lanzar residuos industriales y cloacas domésticas; erosión de costas, laderas, áreas de plantío, terrenos urbanos; alteraciones climáticas que interfieren en el ciclo de las lluvias; urbanización; instalación de hidroeléctricas, entre otras.

Estos factores son históricos y continúan creando problemas; sin embargo, actualmente es posible identificar factores como la presencia de diferentes contaminantes oriundos del proceso del control de plagas y fertilización del suelo, herbicidas y fungicidas que contaminan los manantiales, perjudicando a los usuarios del agua, debemos mencionar también los principios activos de fármacos que al ser excretados por los seres humanos contaminan el medio ambiente.

El avance tecnológico se nos presenta con sus pros y contras; por un lado, contribuye para la producción de nuevos procesos industriales, en contrapartida genera nuevos problemas. En esta perspectiva, podemos identificar los aspectos negativos generados por la propia dinámica de dicho avance tecnológico. Sería la síntesis y la antítesis, ya que los avances generan nuevos problemas que la propia tecnología identifica y concretiza el análisis de estos nuevos conflictos.

Ante esta constatación, el sistema jurídico brasileño posee una estructura apropiada para enfrentar esta cuestión, se identifican en el sistema dos estructuras distintas de control provenientes de políticas públicas que tienen el objetivo común de ofrecer calidad de vida a la población, ambas debidamente apoyadas en principios extraídos de la Constitución Federal Brasileña de 1988. Una de ellas deriva de la Política Nacional del Medio Ambiente, que se fundamenta en el artículo 225, y la otra tiene por base los principios destacados en la sección del capítulo II de la $\mathrm{CF} / 88$.

Ambos sistemas poseen fundamentación específica y son equivalentes en lo que se refiere al control ambiental. Tal política posee competencia 
para proteger, prevenir y mejorar la calidad ambiental, proporcionando beneficios a la calidad de vida de la población brasileña. El sistema de control de la salud, por su parte, trata de identificar y detener las informaciones referentes a los factores ambientales que puedan provocar enfermedades y otros riesgos a la salud.

De este modo es posible identificar la existencia de un acercamiento técnico entre los dos postulados —el medio ambiente y la salud- que se apoyan en los principios de la Constitución Federal de 1988, uno busca la protección del medio ambiente y el otro trata de identificar problemas ambientales que puedan ofrecer riesgos a la calidad de vida, competencias que se equivalen y complementan.

La identificación de la equivalencia entre las competencias levanta una cuestión: ¿es posible identificar si las organizaciones del sistema ambiental y sanitario poseen composiciones distintas actuando sobre el mismo tema? Para encontrar la respuesta, este trabajo analizará el agua como un tema transversal entre los dos sistemas, con la finalidad de evaluar si las organizaciones ambiental y sanitaria poseen composiciones diferentes actuando sobre el mismo objeto.

La intención es realizar la descripción a partir de las normas e identificar las operaciones que tienen vinculación con el objeto del tema de este trabajo, subsistemas debidamente apoyados en la lógica de la teoría de los sistemas sociales, en especial en cuanto al derecho de la sociedad teorizado por Niklas Luhmann. Por lo tanto, al final del capítulo es posible identificar las operaciones y la estructura de cada una de las normas que fueron autorreferenciadas, para entonces realizar la comparación de las operaciones y estructuras e identificar eventuales irritaciones.

\section{ANÁLISIS DE LAS RAMAS DEL DERECHO AMBIENTAL Y SANITARIO}

En este subtítulo, la intención es demostrar la proximidad existente entre las competencias para actuar en la protección del medio ambiente de los subsistemas del derecho ambiental y del derecho sanitario por medio de análisis de los conceptos de diferentes teóricos. La intención es analizar la conceptuación de subsistemas para comprender la relación de las competencias de la actuación.

La teoría de Luhmann sobre los sistemas sociales exige de los nuevos pensadores que sobrepasen la modernidad clásica, con el propósito 
de buscar nuevas categorías, pero en algunos casos las fuentes no fueron trabajadas desde una perspectiva teórica según una concepción corriente, especialmente sobre la historia del derecho. ${ }^{1}$

Sin embargo, es importante entender lo que es derecho. En este contexto, las teorías del derecho, junto con la enseñanza y los textos en vigor, construyen la

...forma por la cual el derecho se presenta como resultado de interpretaciones. De esta forma, comprendido como producto "de auto-observación del sistema jurídico". Sin embargo, en absoluto, no se debe entender el trabajo interno en sistemas jurídicos en la teoría del derecho, en la dogmática jurídica y en los principios y conceptos del derecho, sino en esfuerzos para obtener coherencia conceptual, buscando una verificación de principios, conceptos y reglas. ${ }^{2}$

La teoría jurídica del derecho, a su vez, sería la realización de autodescripción del sistema del derecho, teniendo en cuenta que autoobservaciones y autodescripciones del objeto lo aprehenden sólo si hay distinción de otros objetos. En consecuencia, existe la necesidad de identificación y distinción para poder entonces realizar la asociación. Pero en el contexto actual, lo que se aplica son sólo fórmulas problemáticas como el caso "derecho y sociedad", para pensar que existiría derecho fuera de la sociedad. ${ }^{3}$

El primer concepto analizado es el presentado por Antúnez $z^{4}$ que sostiene que la rama del derecho ambiental, así como las demás ramas de la ciencia jurídica, se apoyan en valores que son el reflejo de la actualidad que expone la propia sobrevivencia del ser humano y que exigen "mantenimiento de las calidades de salubridad del medio ambiente, con la conservación de las especies, la protección de las aguas, del suelo, de la flora, del aire y de todo aquello que es esencial para la vida". ${ }^{5}$

Gracias a esta breve definición es posible percibir que el derecho ambiental es mucho más complejo de lo que parece, una vez que una de sus competencias es asumir una acción de tutela de la protección a la vida de

1 Luhmann, Niklas, O direito da Sociedade, trad. Saulo Krieger, São Paulo, Martin Fontes, 2016, título original: Das Recht des Gesellschaft.

2 Idem.

3 Idem.

4 De Bessa Antunes, Paulo, Direito ambiental, 14a. ed., São Paulo, Atlas, 2012.

5 "Manutenção das qualidades de salubridade do Meio Ambiente, com a conservação das espécies, a proteção das águas, do solo, das florestas, do ar e, enfim, de tudo aquilo que é essencial para a vida" (traducción propia). 
las personas, de especies como la flora y la fauna que sean esenciales para el mantenimiento y garantía del funcionamiento de un sistema dinámico llamado medio ambiente.

Actualmente es imposible disociar la vida en sociedad de la utilización de recursos naturales; todo lo que es utilizado tiene origen a partir de los recursos naturales. El hombre se alimenta, vive, viste, desplaza, cultiva y crea de forma dependiente de los recursos naturales. Esta lógica siempre hizo parte de la humanidad, pero solamente en la actualidad se comprende las funciones del medio ambiente. Por medio de una vision sistémica, es que la gente ha percebido su dependencia del medio ambiente equilibrado.

De esta forma, es posible afirmar que el derecho ambiental puede ser definido como aquel que busca regular la "apropiación económica de los bienes ambientales". Para Antunes, ${ }^{6}$ esta apropiación económica debe llevar en consideración "la sostenibilidad de los recursos, el desarrollo económico y social", para garantizar a los interesados la debida participación en la construcción de las directrices que serán adoptadas, bien como los modelos adecuados de salud y renta.

Ya Michel Prieur ${ }^{7}$ presenta una visión más antropocentrista al afirmar que "el ambiente en el que vivimos es realmente un sinónimo de medio ambiente como sensación arquitectónica y urbana", ${ }^{8}$ pues a través de esta afirmación es posible percibir el intento de incluir el ambiente construido como perteneciente al ramo del derecho ambiental. Prieur asegura que el medio ambiente es un concepto familiar a los ingenieros y urbanistas de la misma forma que lo es para los geógrafos y biólogos. Este medio ambiente abarca todo lo que constituye el territorio del hombre que incluye tanto el ambiente físico como el social.

Prieur ${ }^{9}$ considera que el medio ambiente "es una expresión de interacción y relaciones entre los seres vivos (incluyendo a los humanos), unos con otros y con el ambiente". ${ }^{10} \mathrm{El}$ autor utiliza esta expresión de la existencia de una interacción de las relaciones entre los seres vivos y el medio ambiente para demostrar que la legislación ambiental posee un carácter

6 De Bessa Antunes, Paulo, op. cit.

7 Prieur, Michel. Droit de l'environnment, 5a. ed., París, Dalloz, 2004.

8 "Le cadre de vie est en réalité un synonyme de l'environnement dans son sens architectural et urbanistique", idem.

9 Idem.

10 "Est l'expression des interations et des relations des êtres vivants (dont l'homme), entre eux et avec leur milieu", idem. 
horizontal que abarca diferentes ramos del derecho, sea privado, público o inclusive internacional. Considera que la legislación ambiental, ya sea como derecho de interacción cuya tendencia es incursionar en los diferentes sectores del derecho introduciendo la idea de medio ambiente o demostrando que la protección del medio ambiente debe ser integrada a otras políticas comunitarias.

Prieur llega a la concepción de que la finalidad del derecho ambiental es la búsqueda por un derecho a un ambiente saludable. Afirma que el derecho ambiental no puede estar en una perspectiva puramente positivista del estudio de las reglas jurídicas existentes en ese ámbito, sino debe enfocarse el derecho relativo al medio ambiente.

El derecho ambiental impone límites a la racionalidad del comportamiento ecológico de la sociedad de Massa, que detenta un creciente poder tecnológico, utilizado en la explotación de los recursos naturales, de forma agresiva y predatoria, para alimentar una producción económica ilimitada de bienes de consumo, en un mercado capitalista, que promete calidad de vida basada en la apropiación indiscriminada de riquezas y que desconsidera la naturaliza y su equilibrio como valor relevante. ${ }^{11}$

Es necesario tener claro que el medio ambiente no es el objeto de la ciencia del derecho, pero sí su equilibrio. Mantener el medio ambiente ecológicamente equilibrado es uno de los más complejos e inducen tres desafíos definidos por la CRFB/88, que atribuye la responsabilidad de forma conjunta al Estado y a la sociedad civil, de acuerdo con lo dispuesto en el artículo 225. La propuesta constitucional para una digna y saludable calidad de vida llevó en consideración que el hombre y el medio ambiente forman parte de un mismo contexto ecológico. Por lo tanto, la afectividad de las normas ambientales implica la efectividad de los derechos humanos fundamentales. ${ }^{12}$

La extensión y la complejidad de tales desafíos planteados al derecho constitucional ambiental exigen una postura de diálogo e interacción con todas las demás ciencias que recorren el camino de la temática ambiental, una vez que la búsqueda de la efectividad de las normas ambientales es de suma importancia para el mantenimiento de la vida y la salud en todas sus formas. La vida y la salud como "derechos" no se efectúan sin el equilibrio del medio ambiente.

11 Sueli Padilha, Norma, Fundamentos constitucionais do direito ambiental brasileiro, Río de Janeiro, Elsevier, 2010.

12 Idem.

Esta obra está bajo una Licencia Creative Commons

Atribución-NoComercial-SinDerivar 4.0 Internacional, IIJ-UNAM. 
El derecho ambiental fue revigorizado por el nuevo ropaje constitucional dado al tema del medio ambiente por la Constitución federal de 1988. Corresponde al derecho ambiental actuar sobre toda y cualquier área jurídica que involucra tal temática, imponiendo la reformulación de conceptos, institutos y principios, exigiendo la adaptación y restructuración del modelo socioeconómico actual con el necesario equilibrio del medio ambiente, teniendo en cuenta la calidad de vida saludable. ${ }^{13}$

Por lo tanto, corresponde al derecho ambiental, en cuanto aspecto jurídico en el contexto ambiental, no aislarse en un compartimento estanco, sino respetar y considerar el conocimiento producido en áreas científicas diversas, principalmente la de las ciencias naturales, como la biología, la ecología, la geografía. Con las que no tiene tradición de intercambio; además de las nuevas áreas como el urbanismo, la ingeniería ambiental, la salud pública, cabe también acentuar la comunicación con la economía, la sociología, la antropología, la historia y la filosofía. ${ }^{14}$

En este sentido, Machado ${ }^{15}$ afirma que el derecho ambiental es un derecho sistematizado, que articula la legislación, la doctrina y la jurisprudencia concerniente a los elementos que integran el ambiente. Se busca evitar el aislamiento de los temas ambientales y su enfoque antagónico. No se trata de construir un derecho de las aguas, un derecho de la atmósfera, un derecho del suelo, un derecho forestal, un derecho de la fauna o un derecho de la biodiversidad; el derecho ambiental no ignora lo que cada materia tiene de específico, sino busca interconectar estos temas con el mortero de la identidad de los instrumentos jurídicos de prevención y de reparación, información, monitoreo y participación.

En el mismo sentido, Silva ${ }^{16}$ certifica que la calidad del medio ambiente en que la vivimos, trabajamos y nos divertimos, influye considerablemente en la propia calidad de vida, siendo que la calidad del medio ambiente se transforma, así, en un bien o patrimonio, cuya preservación, recuperación o revitalización se convierten en un imperativo del poder público, para asegurar una buena calidad de vida, que implica en buenas condiciones de

\footnotetext{
13 Idem.

14 Idem.

15 Affonso Leme Machado, Paulo, Direito ambiental brasileiro, 18a. ed., São Paulo, Malheiros, 2010.

16 Afonso Da Silva, José, Direito ambiental constitucional, São Paulo, Malheiros, 2003.
} 
trabajo, ocio, educación, salud, seguridad, en fin, buenas condiciones de bienestar del hombre y su desarrollo.

El problema de la tutela jurídica del medio ambiente se manifiesta a partir del momento en que su degradación pasa a amenazar no sólo el bienestar, sino también la calidad de vida humana y la propia supervivencia del ser humano. De este modo es posible decir que el derecho ambiental trata de ser una disciplina jurídica de acentuada autonomía, dada la naturaleza específica de su objeto — organización de la calidad del medio ambiente con vistas a una buena calidad de vida - , que no se confunde ni se asemeja con el objeto de otras ramas tradicionales del derecho, salvo excepción del derecho sanitario. ${ }^{17}$

Rocha $^{18}$ busca demostrar, por medio del análisis de la legislación infraconstitucional, que el derecho sanitario y el derecho ambiental poseen una importante área en común. Cita la Política Nacional del Medio AmbientePNMA (Ley núm. 6.938/81), en especial sus objetivos de preservación, mejoría y recuperación de la calidad ambiental propicia a la vida, con el objetivo de asegurar la protección de la vida humana, lo que refuerza la conexión entre los dos: salud y medio ambiente.

Del mismo modo, resalta el concepto de contaminación definido en el inciso III del artículo 3o. de la PNMA que, entre las hipótesis de degradación de la calidad ambiental, incluye la contaminación resultante de actividad que directa o indirectamente perjudique la salud, la seguridad y el bienestar de la población o afecten las condiciones estéticas o sanitarias del medio ambiente. ${ }^{19}$

Rocha ${ }^{20}$ destaca que la Ley Orgánica de la Salud — Ley del SUS - (Ley núm. 8.080/90), a su vez, define en su artículo 3o. que la salud tiene como factores determinantes y condicionantes el medio ambiente. La Ley define, en el inciso II del artículo 16, en las letras "a" y "c", como campo de actuación del SUS, la colaboración en la protección al medio ambiente, así como la tarea de participar en la formulación y la implementación de las políticas de control de las agresiones al medio ambiente, de saneamiento básico y aquellas relativas a las condiciones y a los ambientes de trabajo.

\footnotetext{
17 Idem.

18 De Sá Da Rocha, Julio Cesar, Direito da Saúde: direito sanitário na perspectiva dos interesses difusos e coletivos, 2a. ed., São Paulo, LTr, 2011.

19 Idem.

20 Idem

Esta obra está bajo una Licencia Creative Commons

Atribución-NoComercial-SinDerivar 4.0 Internacional, IIJ-UNAM.
} 
Sobre el tema, Rocha ${ }^{21}$ realiza la siguiente constatación: "que la salud y el equilibrio ambiental consagran el efectivo bienestar del ser humano". Ambos derechos — salud y medio ambiente — tienen relación simbiótica, porque enfocan el mismo objeto mediato y se desarrollan sincronizadamente. Si el medio ambiente es degradado, estará afectando de manera directa la salud de aquellos que conviven en ese medio.

Ante tal afirmación no hay cómo alejar la conexión existente entre los temas medioambientales y salud previstos en la CRFB/88 tampoco la equivalencia del derecho sanitario y el derecho ambiental. La relación del medio ambiente y de la salud son "umbilicales", ya que la falta de calidad ambiental puede afectar directa o indirectamente la calidad de la salud de las personas.

En este sentido, Aith ${ }^{22}$ afirma que el derecho sanitario está formado por un conjunto de normas jurídicas que construyen un sistema legal basado en reglas y principios del derecho que orientan y vinculan interpretaciones y decisiones, debidamente apoyadas y sistematizadas a partir de las determinaciones dadas por determinación de la $\mathrm{CRFB} / 88$. En consecuencia, con base en los principios constitucionales, se crearon diversas otras normas jurídicas (leyes, decretos, decretos, resoluciones, etcétera) implementando el sistema que trata y busca practicar la salud en Brasil, debidamente apoyada en un sistema jurídico especializado que apunta a una mejor organización jurídica de las bases determinadas por la Constitución Federal, para el tema de la salud.

La relación del derecho sanitario y del derecho ambiental, en el momento en que procuran actuar en la protección del medio ambiente, acaban teniendo como consecuencia una mejora del ambiente para la convivencia humana y por consiguiente la salud de las personas.

Este tipo de descripción en teoría de los sistemas tiene sentido en la producción de una conexión entre las teorías del derecho y de la sociedad, sobre todo en cuanto a la reflexión del derecho en teoría social. Hay que considerar la transformación de la sociedad estamental en una sociedad moderna, que ocurrió con el auxilio del derecho, principalmente de la revolución ocurrida por medio de la ruptura de la legalidad, comprendida como forma del derecho. Que en la actualidad no es garantía, pues puede generar duda. Aún más si se tienen en cuenta los fenómenos de sobrecarga

21 Idem.

22 Aith, Fernando. Curso de direito sanitário, a proteção do direito à saúde no Brasil, São Paulo, Quartier Latin, 2007. 
del sistema jurídico contemporáneo, que pueden ser resultado "de la acumulación de antiguas reinvindaciones en cuanto a la densidad regulatoria y las nuevas condiciones". Lo que hace que resalte la "dificultad de hacer que la forma del derecho llegue a dar cuenta de los problemas de riesgo o de problemas ecológicos". ${ }^{23}$

Así, de esta forma, la teoría de los sistemas, si es debidamente comprendida, es apta para ejecución de esta tarea, que según Luhmann "es el único candidato provisto de un concepto listo para ejecutar la tarea". Pues según él, "una sociedad compleja sólo puede ser descrita por una sociedad compleja". ${ }^{24}$

\section{Competencias conexas y complementarias identificadas}

en la politica ambiental y de salud

En la práctica es posible identificar algunas acciones y programas que esbozan la necesidad de una actuación en conjunto de los órganos integrantes del Sistema Nacional de Medio Ambiente (Sisnama) y del Sistema Nacional de Vigilancia Ambiental en Salud (Sinvas).

Como referencia se pueden mencionar los programas específicos para el control del agua, suelo, contaminantes y desastres que integran las acciones del Sinvas. Estos programas tienen acciones que priorizan la calidad del recurso natural al que están conectados. En el caso de la Vigilancia Ambiental en Salud relacionada con la Calidad del Agua para Consumo Humano - Vigiagua - el programa defiende que el recurso natural "agua" esté con estándares de calidad que no ofrezcan riesgo a la salud ni a la integridad de las personas. La calidad del agua se puede ver comprometida por diferentes factores, como el lanzamiento de agotamiento sanitario, sin el debido tratamiento, lanzamiento de efluentes de una empresa o incluso un accidente en el transporte de productos peligrosos. En esta situación, tanto los órganos integrantes del Sisnama como de Sinvas poseen competencia para fiscalizar y actuar junto al responsable por el lanzamiento del efluente o transporte de productos.

Sin embargo, si el órgano representante del Sisnama no se comunica con el Sinvas, el problema no puede ser detectado a tiempo para evitar perjuicios a la salud de la población o al medio ambiente. Este ejemplo

\footnotetext{
23 Luhmann, Niklas, op. cit.

24 Idem.
} 
ilustra la necesidad de una actuación conjunta de los órganos integrantes del Sinvas con los del Sisnama y viceversa, ya que ambos poseen la responsabilidad por la ejecución, fiscalización, protección y mejora de la calidad ambiental. El intercambio de información es fundamental para que los programas de estas organizaciones obtengan éxito.

El Sinvas posee programas específicos para el agua, el suelo, las contaminaciones químicas y los desastres, que son los siguientes: Vigiagua, que tiene como objetivo la vigilancia de la calidad del agua para consumo humano; Vigisolo, el cual lleva la vigilancia en salud de poblaciones expuestas a suelo contaminado, que tiene por objetivo desarrollar acciones de vigilancia en salud de poblaciones expuestas a suelo contaminado; Vigipeq se encarga de la vigilancia en salud de poblaciones expuestas a contaminantes químicos, que enfatiza la vigilancia en salud de poblaciones expuestas a contaminantes químicos, y Vigidesastres realiza la vigilancia en salud ambiental relacionada con los riesgos derivados de desastres naturales, que prioriza la vigilancia en salud ambiental de los riesgos asociados a los desastres y su actuación se basa en la gestión de los riesgos.

Para que los programas de Sinvas obtengan los resultados esperados, ellos dependen de comunicación con los integrantes del Sisnama, que deben informar situaciones que puedan comprometer el funcionamiento de los programas. Podrían ser mencionados numerosos ejemplos en que se percibe la importancia de la actuación conjunta, como el lanzamiento de efluentes industriales y residenciales en los manantiales, situaciones de contaminación del suelo, accidentes industriales o de transporte, contaminación del aire, ocupación de áreas de riesgo de deslizamiento, de inundación y ocupación de áreas de preservación permanente.

A partir de leyes de limitación del uso del suelo se percibe una intensa delimitación del uso de áreas de riesgo, que son colocadas en práctica por integrantes del Sisnama que buscan regular el uso y la ocupación de los espacios considerados de riesgo, definidos por la legislación ambiental (en inglés). Esta actuación es próxima a la actuación del Sinvas por medio del programa Vigidesastres, que busca identificar estas áreas y trabajar en el sentido de proteger al ciudadano de problemas que puedan ocurrir y comprometer su salud. En este caso, ambos sistemas podrían actuar de forma conjunta para lograr alcanzar la calidad de vida a través de dos sistemas que tienen diferentes intenciones, pero con objetos comunes. 
Aunque existe la posibilidad de identificar conexiones en la ejecución de las políticas públicas ambientales y de salud, es difícil encontrar actuaciones conjuntas en que realmente las organizaciones integrantes del Sisnama y del Sinvas operen de forma cooperada. De esta forma queda comprobada la relación entre el derecho ambiental y el derecho sanitario, a pesar de que no existe una sintonía en la ejecución de sus competencias. En esta relación se destaca la importancia de investigar la existencia de interferencia entre las competencias de dichos sistemas, así como las condiciones de comunicabilidad entre ellos.

Como ejemplo de la mencionada comunicación deficitaria, se cita el caso de contaminación de un cuerpo hídrico, en que el órgano ambiental integrante del Sisnama toma la iniciativa administrativa y delictiva, sin comunicar a los órganos de salud, representantes del Sinvas para que tomen medidas en el sentido de detectar la posibilidad de que esta contaminación puntual afecte la salud de las personas de esa región o de los sistemas de producción de alimentos. Otro ejemplo sería cuando un órgano de salud, por motivos de seguridad de salud pública, suspende el abastecimiento de agua potable sin buscar identificar los factores responsables de la interrupción, o incluso comunicar al órgano ambiental para que éste identifique a los responsables de la contaminación que provocó la interrupción del servicio de abastecimiento de agua.

En los ejemplos citados es posible constatar que ambas organizaciones deberían haber actuado en el sentido de la protección del medio ambiente y de la salud de las personas, pero en la práctica no siempre ocurre de esta forma, o por desconocimiento de las estructuras y de sus competencias, o por la precariedad de comunicación entre los sistemas en cuanto a la ejecución de sus competencias, o incluso por la falta de conocimiento de la conexión de las competencias entre las organizaciones.

\section{El mapa de normas como tema transversal de actuación del derecho ambiental y sanitario}

Es importante destacar que, para Luhmann, el término "sistemas" no es entendido como una interconexión de ciertas reglas, al contrario de lo que muchos juristas comprenden, sino la interconexión de operaciones factuales que, por medio de la acción de la comunicación operativa, deben ser, sobre todo, comunicaciones, independientemente de lo que éstas afiancen 
en cuanto al derecho. O sea, la distinción entre medio y medio ambiente es el foco de actuación, diferente de otras que buscan el punto de partida en la norma y en la tipología de valores..$^{25}$

Así, es posible identificar una relación sistémica ${ }^{26}$ sobre el tema, donde el agua transita entre las ramas del derecho ambiental y sanitario. En el medio ambiente es posible destacar competencia en lo que se refiere a la protección, la prevención y la mejora de la condición ambiental, condiciones que acaban, consecuentemente, proporcionando beneficios a la calidad de vida de la población brasileña. Para la salud, la actuación busca identificar y detener los riesgos y divulgar las informaciones referentes a los factores ambientales que puedan provocar enfermedades y otros riesgos a la salud.

Para el derecho ambiental, el capítulo del Medio Ambiente de la Constitución federal de 1988 da el debido soporte para la implementación del subsistema jurídico ambiental con el tema agua, de donde es posible destacar las siguientes normas Federales: Política Nacional de Medio Ambiente - Ley 6.938/81; Política Nacional de Recursos Hídricos - Ley 9.433/97; Política Nacional de Saneamiento Básico - Ley 11.445/2007; Política Nacional de Residuos Sólidos - Ley 12.305/10; Política Nacional de Protección y Defensa Civil - Ley 12.608/12; Y la Política Nacional de Protección de la Vegetación nativa - Ley 12.651/12.

\section{TABLA 1. LEGISLACIÓN DE MEDIO AMBIENTE, OBJETO AGUA}

\begin{tabular}{|l|l|}
\hline \multicolumn{1}{|c|}{ Norma } & \multicolumn{1}{c|}{ Lo que dispone } \\
\hline CF/88 & Capítulo de Medio Ambiente \\
\hline Ley 6938/81 & $\begin{array}{l}\text { Dispone sobre la política nacional del medio ambiente, sus fi- } \\
\text { nes y mecanismos de formulación y aplicación, y toma otras } \\
\text { providencias. }\end{array}$ \\
\hline Ley $9.433 / 97$ & $\begin{array}{l}\text { Instituye la política nacional de recursos hídricos, crea el Sis- } \\
\text { tema Nacional de Gerencia de Recursos Hídricos. }\end{array}$ \\
\hline Ley $11.445 / 2007$ & Establece directrices nacionales para el saneamiento básico. \\
\hline Ley 12.305/10 & Instituye la política nacional de residuos sólidos. \\
\hline Ley 12.651/12 & Dispone sobre la protección de la vegetación nativa. \\
\hline
\end{tabular}

25 Luhmann, Niklas, op. cit.
26 Idem. 


\begin{tabular}{|c|l|}
\hline \multicolumn{1}{|c|}{ Norma } & \multicolumn{1}{c|}{ Lo que dispone } \\
\hline Ley 12.608/12 & $\begin{array}{l}\text { Instituye la política nacional de protección y defensa civil } \\
\text { (PNPDEC); dispone sobre el Sistema Nacional de Protección } \\
\text { y Defenza Civil (Sinodec) y el Consejo Nacional de Protec- } \\
\text { ción y Defenza Civil (Cinpdec); autoriza la creación de siste- } \\
\text { ma de informaciones y monitoreo de desastres. }\end{array}$ \\
\hline
\end{tabular}

FUENTE: elaboración propia. ${ }^{27}$

De estas normas es admisible percibir que la actuación privilegia a la protección del medio ambiente y consecuentemente del recurso natural agua, ya que es posible destacar la intención de protección del medio ambiente de forma sistémica. Cada uno con un objetivo más definido, pero partiendo de principios fundamentales del derecho ambiental, debidamente constituidos en la Constitución Federal de 1988.

En cuanto a las normas del derecho sanitario, es posible destacar las siguientes leyes federales: Sistema Único de Salud (Ley 8.080/90); Política Nacional de Recursos Hídricos (Ley 9.433/97); Sistema Nacional de Vigilancia Sanitaria (Ley 9.782/99); Fundación Nacional de Salud (Funasa) (Decreto 7.335/10); Política Nacional de Saneamiento Básico (Ley 11.445/2007), y Política Nacional de Residuos Sólidos (Ley 12.305/10).

En el caso de la salud, la Ley del SUS estructura la actuación administrativa de la salud para construir una base de actuación administrativa que permite, a partir de ella, reconocer la actuación de algunas vigilias (sanitaria, artículo 6o., §1), en la actuación de

...un conjunto de acciones capaces de eliminar, disminuir o prevenir riesgos a la salud y de intervenir en los problemas sanitarios derivados del medio ambiente $[\mathrm{y}]$ que proporcionan el conocimiento, la detección o prevención de cualquier cambio en los factores determinantes y condicionantes de salud individual o colectiva. ${ }^{28}$

27 Cardoso Neto, Nicolau, Água com qualidade para o consumo humano: normas e sobreposição de competências entre o direito ambiental e sanitário, Río de Janeiro, Lumen Juris, 2018, p. 165.

28 Brasil. Lei no. 8.080, de 19 de setembro de 1990, "Dispõe sobre as condições para a promoção, proteção e recuperação da saúde, a organização e o funcionamento dos serviços correspondentes e dá outras providências, Diário Oficial da República Federativa do Brasil, Brasília, DF, 20 de setembro de 1990, disponible en: http://www.planalto.gov. br/ccivil_03/leis/L8080.htm.

Esta obra está bajo una Licencia Creative Commons

Atribución-NoComercial-SinDerivar 4.0 Internacional, IIJ-UNAM. 


\section{TABLA 2. LEGISLACIÓN DE SALUD, OBJETO AGUA}

\begin{tabular}{|l|l|}
\hline \multicolumn{1}{|c|}{ Norma } & \multicolumn{1}{c|}{ Lo que dipone } \\
\hline LF/88 & $\begin{array}{l}\text { Sección de la salud } \\
\text { Dispone sobre las condiciones para la promoción, protec- } \\
\text { ción y recuperación de la salud, la organización y el fun- } \\
\text { cionamiento de los servicios correspondientes y toma otras } \\
\text { providencias }\end{array}$ \\
\hline Ley 9.433/97 & $\begin{array}{l}\text { Instituye la política nacional de recursos hídricos, crea el } \\
\text { Sistema Nacional de Gerencia de Recursos Hídricos } \\
\text { Define el Sistema Nacional de Vigilancia Sanitaria, crea la } \\
\text { Agencia Nacional de Vigilancia Sanitaria y toma otras pro- } \\
\text { videncias. }\end{array}$ \\
\hline Decreto 7.335/10 & $\begin{array}{l}\text { Aprueba el Estatuto y el Cuadro Demostrativo de los Cargos } \\
\text { en Comisión y de las Funciones Gratificadas de la Funda- } \\
\text { ción Nacional de Salud-Funasa, y toma otras providencias. }\end{array}$ \\
\hline Ley 11.445/2007 & $\begin{array}{l}\text { Establece directrices nacionales para el saneamiento básico. } \\
\text { Instituye la política nacional de residuos sólidos. }\end{array}$ \\
\hline Ley 12305/10 &
\end{tabular}

FUENTE: elaboración propia. ${ }^{29}$

A través de toda esta estructura, el derecho sanitario acaba constituyendo un sistema capaz de identificar y detener los riesgos y divulgar las informaciones referentes a los factores ambientales que puedan provocar enfermedades y otros agravios a la salud. Para el objeto agua, el Ministerio de Salud instituyó portaría de potabilidad de agua (Portaría MS núm. 2914 del 12 de diciembre de 2011), con la intención de instruir un parámetro mínimo de seguridad. el derecho ambiental, busca instituir por medio de dos grandes políticas (medio ambiente y de recursos hídricos) la protección y la prevención de la calidad ambiental.

\section{CONCLUSIÓN}

A partir de la investigación realizada se verificó que las ramas del derecho ambiental y del derecho sanitario poseen fundamentos equivalentes en cuanto al agua como tema transversal de actuación. A través de la investigación realizada sobre el subsistema derecho ambiental se destacan

29 Cardoso Neto, Nicolau, op. cit., p. 229.

Esta obra está bajo una Licencia Creative Commons Atribución-NoComercial-SinDerivar 4.0 Internacional, IIJ-UNAM. 
las medidas de protección, prevención y de mejora de la calidad ambiental como requisitos para garantizar que todos tengan derecho al medio ambiente ecológicamente equilibrado, balance necesario para el mantenimiento del agua con calidad y cantidad suficiente para los diferentes usos.

En cuanto al subsistema del derecho sanitario, el foco de actuación es identificar y detener los riesgos y divulgar las informaciones referentes a los factores ambientales, relacionados con el agua, que puedan provocar enfermedades y otros agravios a la salud, sin que sea necesario para la realización de acciones de protección y preservación ambiental. De esta comprobación es posible afirmar que ambas ramas actúan sobre el medio ambiente con la finalidad, directa o indirecta, de defender la calidad de vida de las personas.

Por lo tanto, fue posible identificar la existencia de una proximidad técnica entre los dos subsistemas de la ciencia jurídica, que se amparan en principios y competencias constitucionales distintas de la Constitución federal de 1988, ya que el derecho ambiental apunta a la protección del medio ambiente, que ofrece como resultado calidad de vida para las personas y el derecho sanitario que busca identificar problemas ambientales que puedan ofrecer perjuicios a la calidad de vida de la población. De este modo se puede afirmar que, estas ramas, a pesar de ser instituidas por objetos diferentes, medio ambiente y salud, poseen competencias equivalentes y complementarias.

Por medio de esta constatación se responde la cuestión de esta investigación que trató sobre la posibilidad de identificar si: ¿las organizaciones del sistema ambiental y sanitario poseen composiciones distintas actuando sobre el mismo tema?

Para responder el problema de la investigación este trabajo analizó el agua, como tema transversal de actuación de ambos subsistemas, a fin de evaluar si las organizaciones ambiental y sanitaria poseen composiciones distintas actuando sobre el mismo objeto.

Esta relación entre el medio ambiente y la salud es perceptible en la Constitución Federal/88, en especial en los artículos 200 y 225, lo que admite identificar su correspondencia en cuanto al objeto de proposición de calidad de vida para la población brasileña, a pesar de estar expuestos en diversos capítulos de la Constitución.

Luhmann llama la atención sobre el hecho de que el autorreferir caracteriza el sistema como objeto y no como concepto, lo que posibilita afirmar 
que el sistema es diferenciado de todos los demás y no sólo de determinados. Esto admite garantizar que el sistema se caracteriza no como sistema solo, sea social, del derecho, económico, sino como diferencia de otros que realizan su propia autodesignación, independientemente de lo que ocurra. El acoplamiento estructural sería un contraste aquéllos, una vez que éste ocurre en el momento en que el sistema supone características determinadas de su ambiente, de forma que las utiliza estructuralmente, considerando que lo que incluyó es tan importante como lo que excluyó, lo que es el acoplamiento. Siendo las configuraciones de acoplamientos estructurales restrictivos, de forma que facilitan la influencia del ambiente sobre el sistema.

\section{BIBLIOGRAFÍA}

Affonso Leme Machado, Paulo, Direito ambiental brasileiro, 18a. ed., São Paulo, Malheiros, 2010.

Afonso DA Silva, José, Direito ambiental constitucional, São Paulo, Malheiros, 2003.

AITH, Fernando, Curso de direito sanitário. A proteção do direito à saúde no Brasil, São Paulo, Quartier Latin, 2007.

Bessa Antunes, Paulo de, Direito ambiental, 14a. ed., São Paulo, Atlas, 2012.

CARdoso Neto, Nicolau, Água com qualidade para o consumo humano: normas e sobreposição de competências entre o direito ambiental e sanitário, Río de Janeiro, Lumen Juris, 2018.

Luhmann, Niklas, $O$ direito da Sociedade, trad. de Saulo Krieger, São Paulo, Martin Fontes, 2016 (título original: Das Recht des Gesellschaft).

PRIEUR, Michel, Droit de l'environnment, 5a. ed., París, Dalloz, 2004.

SÁ DA RochA, Julio César de, Direito da saúde: direito sanitário na perspectiva dos interesses difusos e coletivos, 2a. ed., São Paulo, LTr, 2011.

Sueli PADILHA, Norma, Fundamentos constitucionais do direito ambiental brasileiro, Río de Janeiro, Elsevier, 2010.

Fecha de recepción: 19 de julio de 2017.

Fecha de aceptación: 30 de mayo de 2019. 